\title{
Neurodegenerative diseases: challenges
}

\author{
Vinod Labhasetwar
}

Published online: 7 September 2011

(C) Controlled Release Society 2011

Diseases of the aging brain-including Alzheimer's, Parkinson's, and Huntington's - affect the lives of millions and pose a growing threat to public health. These diseases occur as a result of neurodegenerative processes involving the progressive loss of structure or function of neurons, including death of neurons. The population of the USA is aging, and an ever-increasing number of Americans are afflicted with neurodegenerative diseases. It is estimated that approximately one in four Americans will suffer from a neurodegenerative disease, and virtually all Americans will have a family member with one of these conditions. According to the World Health Organization's estimate, there are currently about 18 million people worldwide with Alzheimer's disease alone, and this figure is projected to nearly double by 2025 .

The precise pathophysiological mechanisms underlying neurodegenerative disorders - and how they lead to diseaseare not well understood. The most common understanding is the increased level of oxidative stress with aging. However, there is a vast amount of evidence indicating that neurotrophic factors (NTFs) play a major role in the development, maintenance, and survival of neurons [1]. It is therefore hypothesized that NTFs might be used to treat human neurodegenerative diseases. One of the significant challenges in their potential clinical use is the effective delivery of NTFs to the central nervous system (CNS). NTFs are large-molecular-weight proteins, unstable in the body because of rapid degradation because of proteases and unable to cross the blood-brain barrier. To circumvent these

V. Labhasetwar $(\square)$

Department of Biomedical Engineering/ND20, Lerner Research Institute, Cleveland Clinic,

Cleveland, OH 44195, USA

e-mail: labhasv@ccf.org issues, direct intracerebroventricular injection/infusion of NTFs has been tested. In this regard, sustained-release biodegradable microspheres [2] or scaffolds loaded with NTFs implanted in the brain have been investigated. One recent approach includes implanting stem cells with scaffold that can deliver NTFs to promote differentiation of stem cells into neurons [3]. In addition, several viral vectors, including adenovirus, adeno-associated viral vectors, and lentiviruses, have been tested [4].

The article by Herzog et al. in this issue of the journal presents data with adeno-associated viral vector, expressing one of two different NTFs: nerve growth factor (NGF) and neurturin (NRTN). The vectors were tested via direct brain injection. The data demonstrated: (1) 2-year, targeted, bioactive protein in monkeys, (2) persistent, bioactive protein throughout the life span of the rat, and (3) accurately targeted bioactive protein in aged rats, with (4) no safety issues or antibodies to the protein detected. The long-term expression of therapeutic protein in the brain is critical to combat chronic neurodegenerative diseases. The results of these studies provide empirical guidance by which to establish parameters for human dosing and collectively support the idea that gene transfer may overcome key delivery obstacles that have precluded successful translation of neurotrophic factors to the clinic. The AAV2-NGF and AAV2-NRTN vectors are now being tested in patients with Alzheimer's and Parkinson's disease, respectively, as a part of ongoing phase 2 clinical trials.

In its strategic plan, "Neuroscience in the New Millennium," the National Institute of Neurological Disorders and Stroke (NINDS) of the National Institutes of Health has pointed out that many neurological disorders will become more frequent as the US population ages. As part of its strategic plan, the NINDS hopes to invest across the full 
spectrum of basic, translational, and clinical research. Basic research is essential to understanding the causes and pathways involved in neurodegenerative diseases. Interventions such as deep brain stimulation (DBS), which involves the implantation of a medical device called a brain pacemaker that sends electrical impulses to specific parts of the brain, are now being used to treat a variety of disabling neurological symptoms - most commonly, the debilitating symptoms of Parkinson's disease, such as tremor, rigidity, stiffness, slowed movement, and walking problems [5]. Although DBS is helpful for some patients, there is also the potential for unintended neuropsychiatric side effects, and patients still may need to be on medication. Thus, a combined effort involving basic and translational research is needed to combat the growing epidemic of neurodegenerative diseases.

\section{References}

1. Levy YS, Gilgun-Sherki Y, Melamed E, Offen D. Therapeutic potential of neurotrophic factors in neurodegenerative diseases. BioDrugs. 2005;19(2):97-127.

2. Menei P, Pean JM, Nerriere-Daguin V, Jollivet C, Brachet P, Benoit JP. Intracerebral implantation of NGF-releasing biodegradable microspheres protects striatum against excitotoxic damage. Exp Neurol. 2000;161(1):259-72.

3. Xiong Y, Zeng YS, Zeng CG, Du BL, He LM, Quan DP, et al. Synaptic transmission of neural stem cells seeded in 3-dimensional PLGA scaffolds. Biomaterials. 2009;30(22):3711-22.

4. Davidson BL, Breakefield XO. Viral vectors for gene delivery to the nervous system. Nat Rev Neurosci. 2003;4(5):353-64.

5. Alberts JL, Hallahan K, Thota A, Noecker AM, Vitek JL, McIntyre CC. Reducing cognitive-motor declines associated with bilateral subthalamic deep brain stimulation through computational modelling in a Parkinson's disease patient. J Neurol Neurosurg Psychiatry. 2010;81(10):1170-2. 